\title{
Control robusto con QFT del pH en un fotobiorreactor raceway
}

\author{
Ángeles Hoyo, José Luis Guzmán, José Carlos Moreno, Manuel Berenguel \\ Dep. de Informática, Universidad de Almería, 04120 Almería, España. Tel: +34 950 214133; \\ \{ahs715,joseluis.guzman,jcmoreno,beren\}@ual.es
}

\section{Resumen}

Este trabajo presenta una primera aproximación al control robusto del $\mathrm{pH}$ en un fotobiorreactor raceway para el cultivo de microalgas. En este tipo de procesos el $p H$ es la variable más crítica a controlar teniendo una relación directa con la productividad del sistema. La dinámica del pH posee un carácter fuertemente no lineal estando afectada por numerosos factores tales como el aporte del $\mathrm{CO}_{2}$ al medio de cultivo o la realización de la fotosíntesis por parte de las microalgas. Esta no linealidad genera una gran fuente de incertidumbre en el proceso incluso cuando el sistema es controlado alrededor del punto de operación deseado. Por tanto, en este artículo se ha procedido a realizar el modelado del sistema con incertidumbre paramétrica cubriendo los rangos de trabajo típicos del $p H, y$ posteriormente se ha realizado el diseño de un controlador robusto con QFT para conseguir unos requisitos de rendimiento y estabilidad robustos determinados. El algoritmo de control resultante se ha evaluado en simulación frente a distintas condiciones de trabajo y en distintos puntos de operación obteniéndose resultados satisfactorios.

Palabras clave: control robusto, QFT, fotobiorreactores, simulación.

\section{Introducción}

Los fotobiorreactores raceway han sido estudiados desde 1950 con el fin de aportar una solución para el cultivo de microalgas a escala industrial. Hoy en día, debido a su escalabilidad y su factibilidad, son considerados como la tecnología más adecuada para el cultivo industrial de micoralgas. La popularidad de los reactores raceway viene dada de su bajo coste de inversión inicial cuando son comparados con otras tecnologías, como los fotobiorreactores tubulares, los cuales requieren estructuras y equipos más complejos. Otras ventajas relevantes del reactor raceway son la simplicidad de operación y sus bajos costes de mantenimiento [1].

Numerosos estudios se han centrado en la selección óptima del diseño y configuración de los fotobio- rreactores raceway para conseguir que las microalgas crezcan en las mejores condiciones. Actualmente, existen numerosos diseños de fotobiorreactores raceway que varían ligeramente del diseño original propuesto por Oswald y Golueke [2], el cual aseguraba las condiciones óptimas de crecimiento. A pesar de la gran cantidad de diseños de la estructura del fotobiorreactor, el trabajo principal es el mismo y una gran cantidad de factores similares deben de ser alcanzados para obtener la producción deseada de biomasa, todos ellos relacionados con las variables que influyen en el proceso de crecimiento [3].

Tal y como se indica en [4], las variables más importantes que afectan al crecimiento de las microalgas son temperatura del medio, radiación solar, pH y oxígeno disuelto. Además, la respuesta fotosintética de las microalgas a la radiación solar depende de otras muchas variables que hacen que el sistema sea extremadamente complejo [5]. Para los reactores raceway, los requisitos de incidencia de la radiación solar y las condiciones de operación de temperatura viene determinados generalmente por la propia arquitectura del sistema. Por tanto, las variables a controlar en este tipo de reactores son el pH y el oxígeno disuelto. Ambas variables tienen una dinámica altamente dependiente del proceso de fotosíntesis y es necesario mantenerlas cercanas a unos puntos de operación deseados [6]. De estas dos variables, el pH es la variable más importante a controlar ya que posee una influencia directa en la correcta realización de la fotosíntesis. Dicha variable presenta una dinámica fuertemente no lineal viéndose afectada por la inyección de $\mathrm{CO}_{2}$ en el medio y por el consumo de $\mathrm{CO}_{2}$ durante el proceso de fotosíntesis. El control de esta variable se ha llevado a cabo en la literatura desde distintos puntos de vista. En [7] se realizó un control lineal con PI más controlador por adelanto en torno al punto de operación deseado. Por otro lado, en [6] se realizó un control basado en eventos utilizando control predictivo basado en modelo, que posteriormente fue mejorado y combinado con un control selectivo y simultáneo del oxígeno disuelto [8].

Este trabajo presenta una nueva solución para el 
control de pH mediante un diseño de un controlador robusto con QFT. De esta forma, la dinámica no lineal del sistema, así como las distintas fuentes de error, se han capturado en forma de un modelo de bajo orden con incertidumbre paramétrica. Una vez obtenido dicho modelo, se ha procedido a diseñar un controlador PID robusto con el fin de cumplir unas especificaciones determinadas de rendimiento y estabilidad. Los resultados han sido evaluados satisfactoriamente en un simulador no lineal del proceso [5], analizando el comportamiento en distintos puntos de operación y bajo diferentes condiciones de trabajo externas.

En la sección 2 del artículo se mostrará un breve resumen del proceso real del cual se han obtenido los datos utilizados en este trabajo. El modelo del sistema será presentado en la sección 3, y la sección 4 se dedica al diseño del controlador robusto con QFT. Los resultados de simulación se muestran en la sección 5, y la sección 6 está dedicada a conclusiones y futuros trabajos.

\section{Descripción del proceso}

El fotobiorreactor raceway utilizado como referencia en este proyecto y del cual se han obtenido los datos utilizados en este artículo se encuentra en la Estación Experimental Las Palmerillas, perteneciente a la fundación CAJAMAR (Almería). El fotobiorreactor tiene una área superficial de un total de $100 \mathrm{~m}^{2}$, formado por dos canales de $50 \mathrm{~m}$, cada uno de $1 \mathrm{~m}$ de ancho, unidos mediante una curva en forma de U (ver Figura 1). El fotobiorreactor trabaja a una profundidad constante de $0.2 \mathrm{~m}$, tal y como recomienda Mendoza [9] para dar las mejores condiciones hidráulicas en términos de consumo de potencia, aportando un volumen total al reactor de $20 \mathrm{~m}^{3}$.

La parte de mezcla está formada por una rueda de pedales de $1.2 \mathrm{~m}$ de diámetro con 8 palas, hechas de contrachapado y controladas por un motor eléctrico (W12 35 kW, 1500 rpm, Ebarba, Barceona) con engranajes reductores.

La carbonación se produce a un 1 de profundidad, en una fosa de $0.65 \mathrm{~m}$ de ancho, que abarca el ancho total del canal $(1 \mathrm{~m})$, y está situada a 1.8 $\mathrm{m}$ aguas debajo de las palas. El flujo de gas es inyectado mediante una membrana difusora de 3 platos en la parte más baja del foso. La pista y el foso están hechas de fibra de vidrio blanca de 3 mm de ancho (ver Figura 2).

\section{Modelado del proceso}

Desde el punto teórico, el control del pH es un problema no lineal, que solo puede ser linealizado

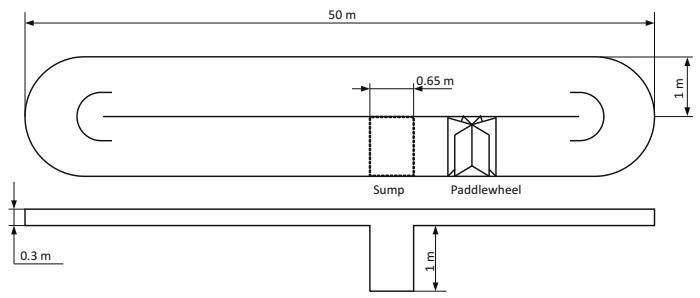

Figura 1: Esquema del fotobiorreactor

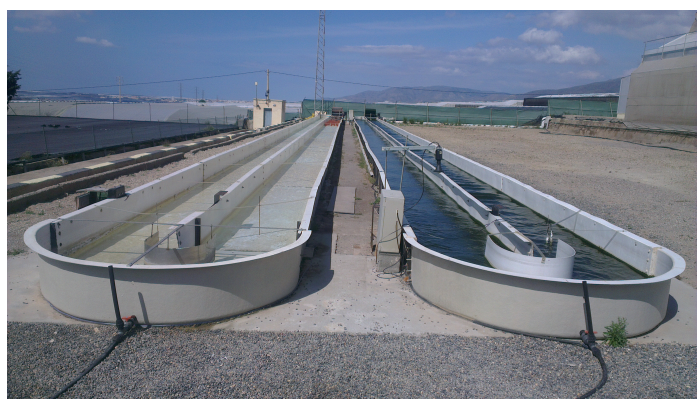

Figura 2: Fotobiorreactor industrial raceway

bajo varias circunstancias [5]. Considerando que la salida del proceso es el pH del cultivo, la apertura de la válvula de inyección de $\mathrm{CO}_{2}$ es la variable manipulable, y la radiación solar es la mayor perturbación del sistema, el comportamiento del sistema puede ser representado con la siguiente ecuación diferencial [10], [11]:

$$
\tau \frac{d p H}{d t}=u\left(t-t_{r}\right)+\beta\left(p H_{0}-p H\right)+\gamma p H
$$

El $p H_{0}$ es el pH en el punto de equilibro, $\beta$ es un factor para ajustar la contante de tiempo del sistema, $\alpha$ es la ganancia estática del sistema, $u$ es la señal de control, que representa las inyecciones de $\mathrm{CO}_{2}, t_{r}$ es el tiempo de retardo, y $\gamma$ and $\tau$ son los parámetros que dependen de la radiación solar y del estado del cultivo, respectivamente.

Este modelo lineal de bajo orden ha sido identificado teniendo en cuenta la estructura del fotobiorreactor, la distribución del sensor y actuador y las dinámicas observadas en los datos. El modelo anterior relacionado con la salida del pH y la inyección de $\mathrm{CO}_{2}$ puede ser representada con las siguientes funciones de transferencia [10]:

$$
p H=\frac{k}{1+\tau s} e^{-t_{r} s} u_{C O_{2}}+\frac{k_{r}}{1+\tau_{r} s} I
$$

donde $p H$ es el pH del cultivo, $u_{C_{2}}$ es el porcentaje de apertura de la válvula, $I$ es la radiación global, $k$ y $k_{r}$ son las ganancias estáticas, y $\tau$ y $\tau_{r}$ las constantes de tiempo correspondientes. 
Los modelos utilizados en este artículo para el desarrollo del control robusto has sido obtenidos para diferentes puntos de operación en torno a los cuales la productividad del sistema es máxima. Para ello, se han analizado los datos de relación $\mathrm{pH}$ y productividad observados en la figura 3 [8].

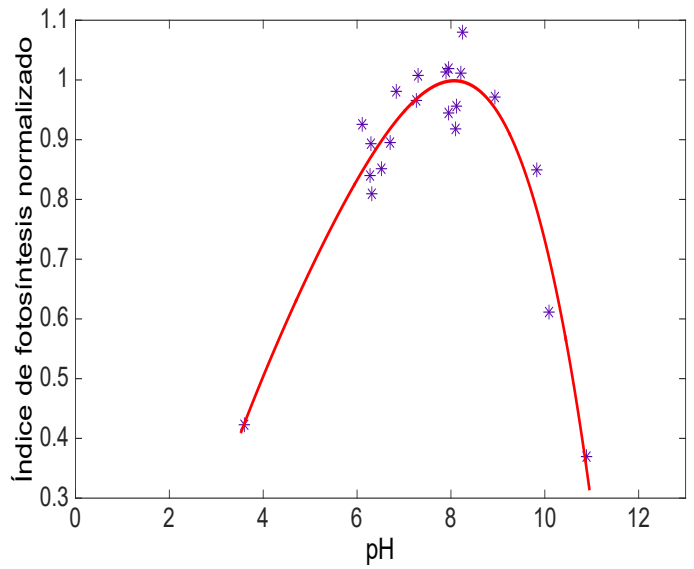

Figura 3: Relación pH y productividad

De esta forma, se escogieron los puntos de operación $\mathrm{pH}=7, \mathrm{pH}=8$ y $\mathrm{pH}=9$, cubriendo así un amplio rango de trabajo y donde, como se verá más adelante, el modelo varía tanto en ganancia, en constante de tiempo y en retardo.

Para obtener los modelos, se han realizado diferentes trenes de pulsos en la inyección del $\mathrm{CO}_{2}$ a lo largo del periodo diurno y en torno a cada uno de los puntos de operación, para un total de 20 días con diferentes condiciones medioambientales. Los modelos obtenidos se basan en la función de transferencia de primer orden del primer término de la ecuación (2). Destacar que, en esta primera versión del diseño de control robusto, únicamente se ha obtenido el modelo de esta primera función de transferencia, dejando pendiente el cálculo de la incertidumbre del segundo término para un trabajo futuro. La figura 4 muestra un ejemplo la validación del modelo para un determinado día de ensayo.

Se hicieron ensayos de 20 días y 3 puntos de operación diferentes, ascendiendo a un total de 60 ensayos. Una vez obtenidos los modelos para cada uno de estos ensayos, se calcularon las cotas de incertidumbre para el modelo del $\mathrm{pH}$, resultando en (constantes de tiempo y retardo en segundos):

$$
\begin{aligned}
p H & =\frac{k}{1+\tau s} e^{-t_{r} s} C O_{2} \\
k & =[-4,1,-0,35] \\
\tau & =[3,4,8] \cdot 10^{3} \\
t_{r} & =[1,1,4] \cdot 10^{2}
\end{aligned}
$$

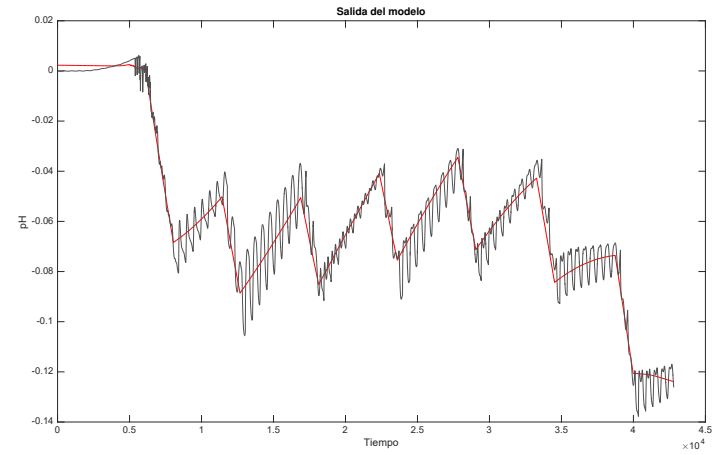

Figura 4: Validación del modelo. En rojo el modelo resultante.

Destacar la gran variabilidad en los parámetros debido al efecto diurno de la radiación en el proceso de fotosíntesis y el cambio de punto operación.

\section{Diseño del controlador}

Teniendo en cuenta el modelo de primer orden con retardo comentado en el apartado anterior, y los valores de incertidumbre para ganancia, constante de tiempo y retardo, se ha optado por diseñar un controlador robusto basado en QFT [12].

Se asumen unas especificaciones de estabilidad dadas por un margen de fase mayor o igual a 45 grados para todos los puntos de operación. Para el seguimiento de referencias se han tomado las cotas dadas por las siguientes ecuaciones:

$$
\begin{gathered}
T_{l}(s)=\frac{1}{(6400 s+1)(2600 s+1)(1000 s+1)} \\
T_{u}(s)=\frac{1}{3500 s+1}
\end{gathered}
$$

Estas especificaciones en frecuencia se corresponden con una constante de tiempo de lazo cerrado entre 58 y 175 minutos.

El conjunto de frecuencias seleccionado para diseño ha sido $W=\{0,0001,0,0005,0,001,0,01\}$ rad/s. La figura 5 muestra la representación de la incertidumbre en el plano de Nichols para las frecuencias en $W$. En cada plantilla el nominal, dado por la ecuación (6), es marcado con un asterisco.

$$
P_{0}(s)=\frac{-4,1}{3390 s+1} e^{-100 s}
$$

En la figura 6 se muestran las fronteras correspondientes a la especificación de estabilidad de $2.32 \mathrm{~dB}$ y a la especificación de seguimiento de referencias dada por la magnitud de las cotas en las ecuaciones (4) y (5), evaluadas para las frecuencias en 


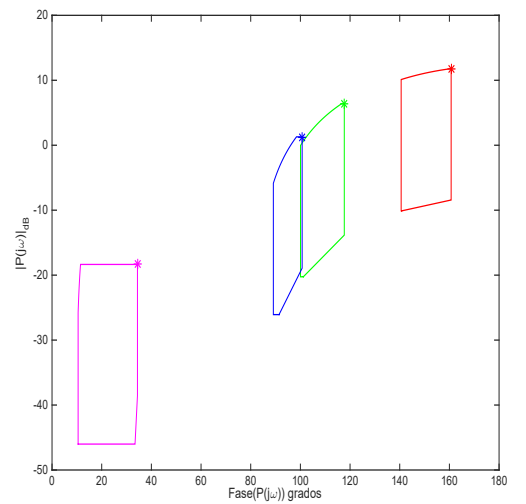

Figura 5: Plantillas (0.0001 - rojo, 0.0005 - verde, 0.001 - azul, 0.01 - magenta).

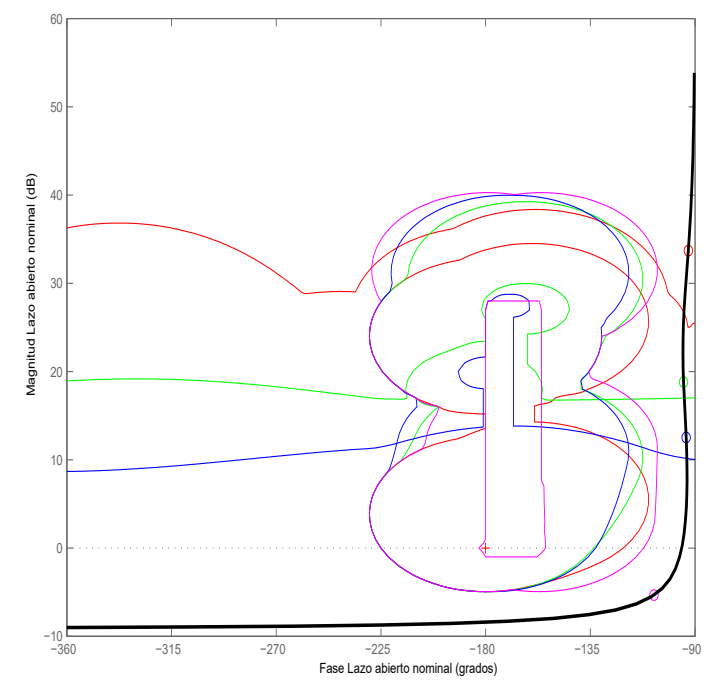

Figura 6: Ajuste de $L_{0}$, fronteras de estabilidad y seguimiento (0.0001 - rojo, 0.0005 - verde, 0.001 azul, 0.01 - magenta).

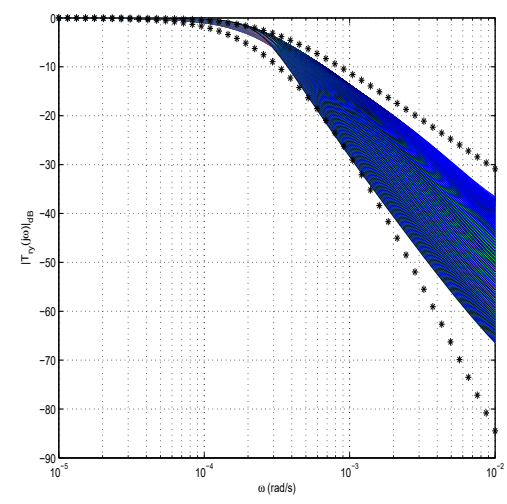

Figura 7: Validación de las especificaciones de seguimiento de referencia.

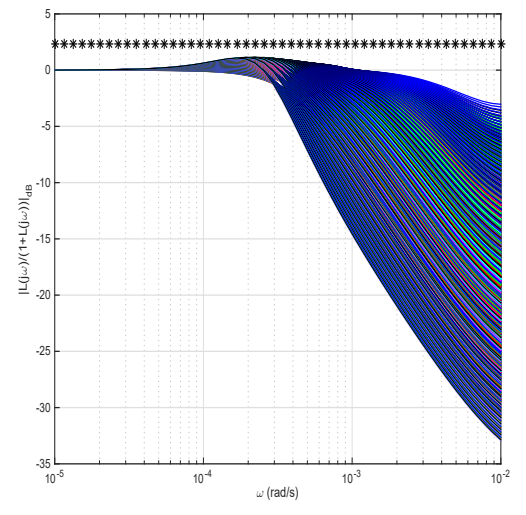

Figura 8: Validación de las especificaciones de estabilidad.

el conjunto $W$. En esta figura se puede observar también el ajuste de la función nominal de lazo abierto para el controlador PID dado por la siguiente ecuación:

$$
C(s)=-34,2852\left(1+\frac{0,00035}{s}\right)(1+83,89265 s)
$$

Las figuras 7 y 8 muestran la validación del diseño para las especificaciones de seguimiento y de estabilidad, respectivamente.

\section{Resultados}

Esta sección presenta la evaluación en simulación del controlador robusto diseño en los distintos puntos de trabajo. Para evaluar su correcto funcionamiento se han tomado como referencia diversos días con distintas condiciones climatológicas y en distintos puntos de operación, tratando de evaluar el rendimiento del controlador frente a la variabilidad de la dinámica del proceso.

Las simulaciones se han llevado a cabo haciendo uso del modelo no lineal del fotobiorreactor implementado en Simulink y desarrollado en [5]. Con el fin de analizar el comportamiento del sistema en los diversos puntos de trabajo, como referencia se le ha introducido, en diferentes ensayaos, escalones descendientes del pH al comienzo del día.

Las figuras 9, 10, 11 y 12 muestran dos ensayos de ejemplo para un día soleado y otro con nubes, cada uno de ellos además en distintos puntos de operación, comenzando los escalones de los ensayos en valores iniciales de $\mathrm{pH}$ de 8.3 y 7.9 respectivamente. En dichas figuras se puede observar que el comportamiento del sistema es acorde a las especificaciones de diseño y es prácticamente el mismo en ambos días a pesar de comenzar en distintos puntos de trabajo y estar sometido a diferentes condiciones de clima, lo que hace demostrar el correcto funcionamiento del controlador robusto propues- 

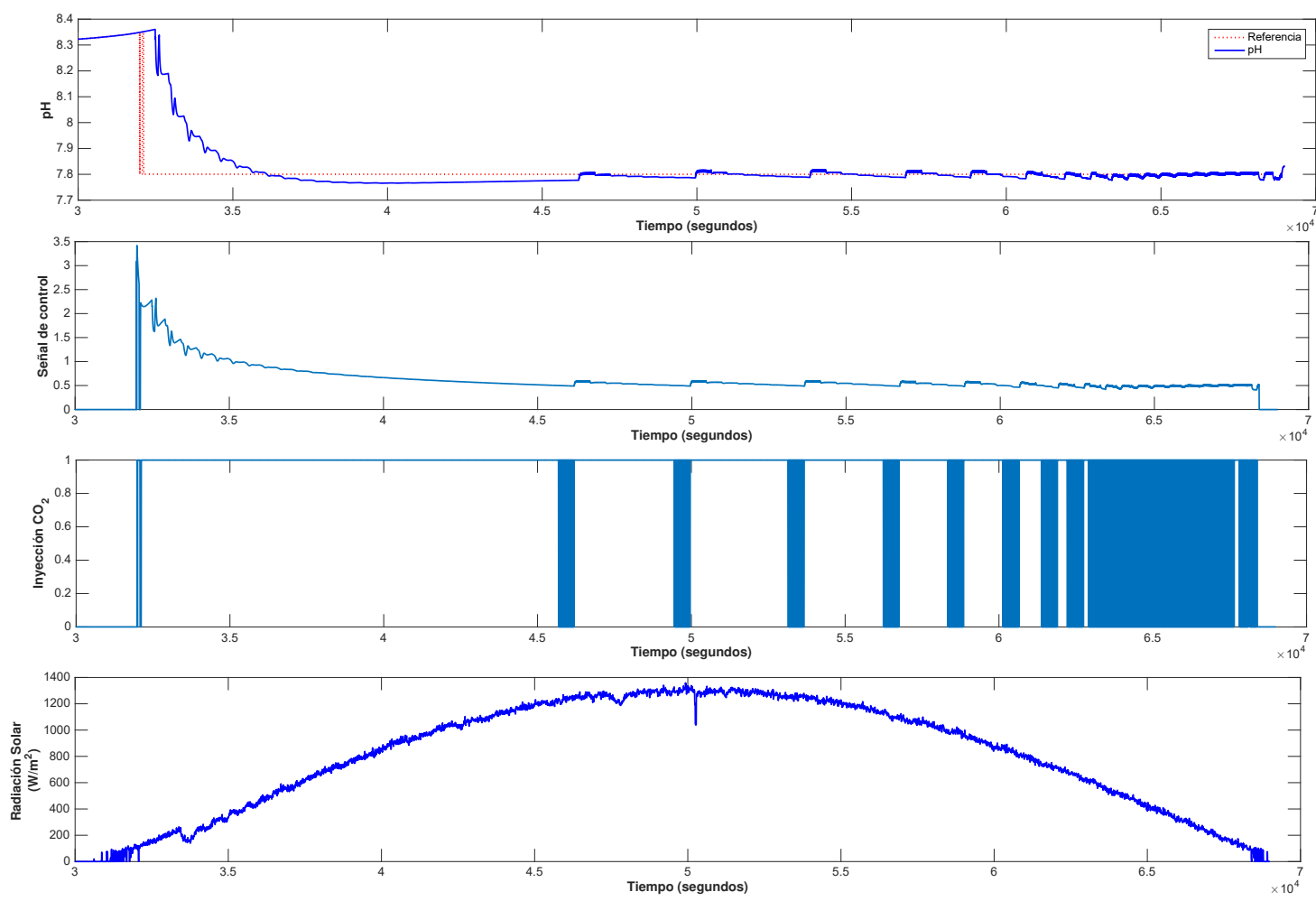

Figura 9: Ensayo día soleado para para un valor inicial de $\mathrm{pH}=8.3$
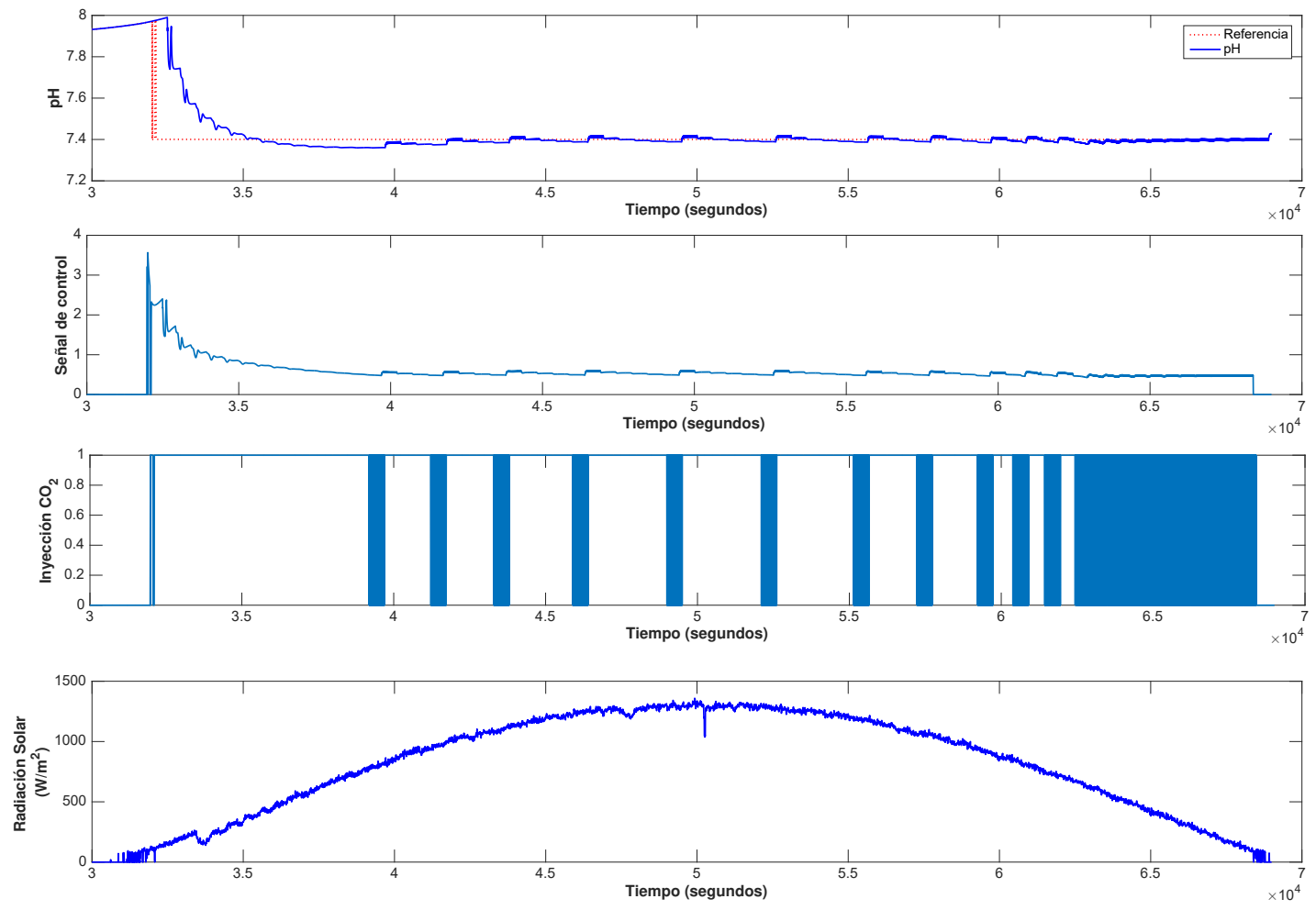

Figura 10: Ensayo día soleado para para un valor inicial de $\mathrm{pH}=7.9$ 

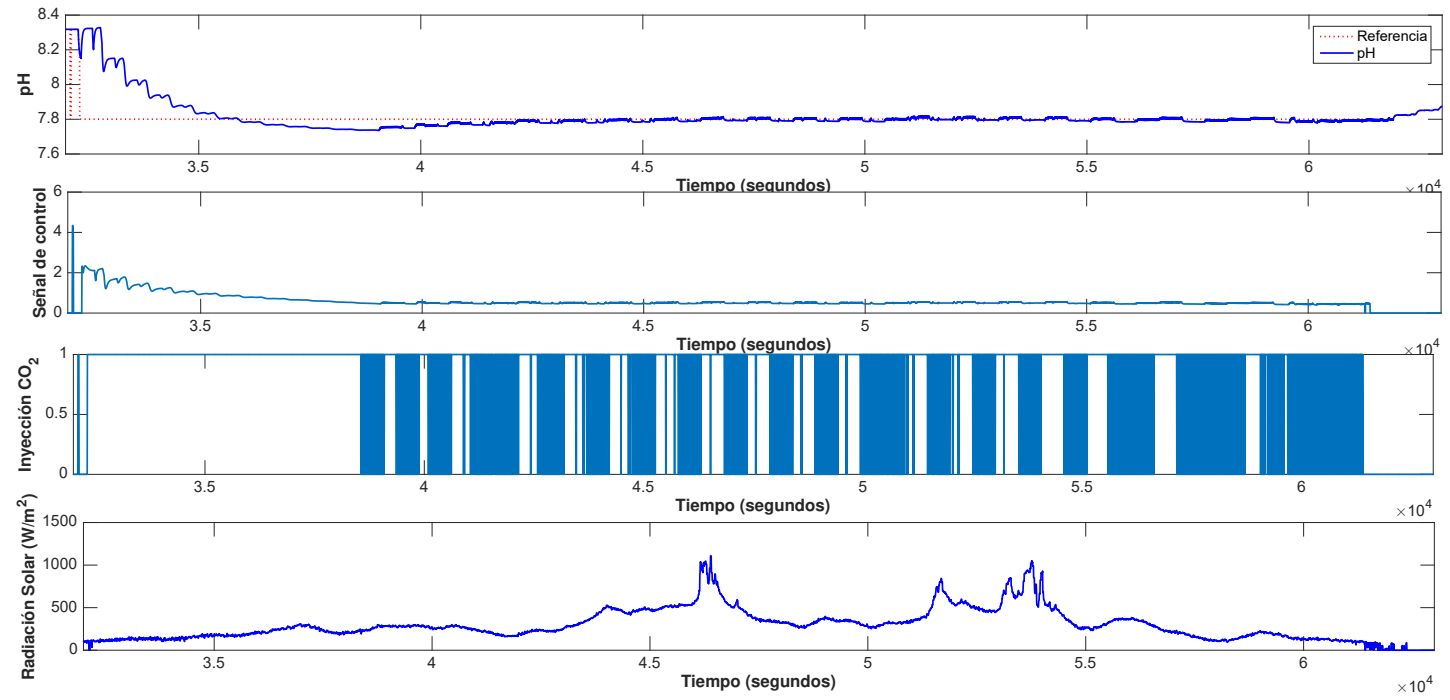

Figura 11: Ensayo día nublado para para un valor inicial de $\mathrm{pH}=8.3$
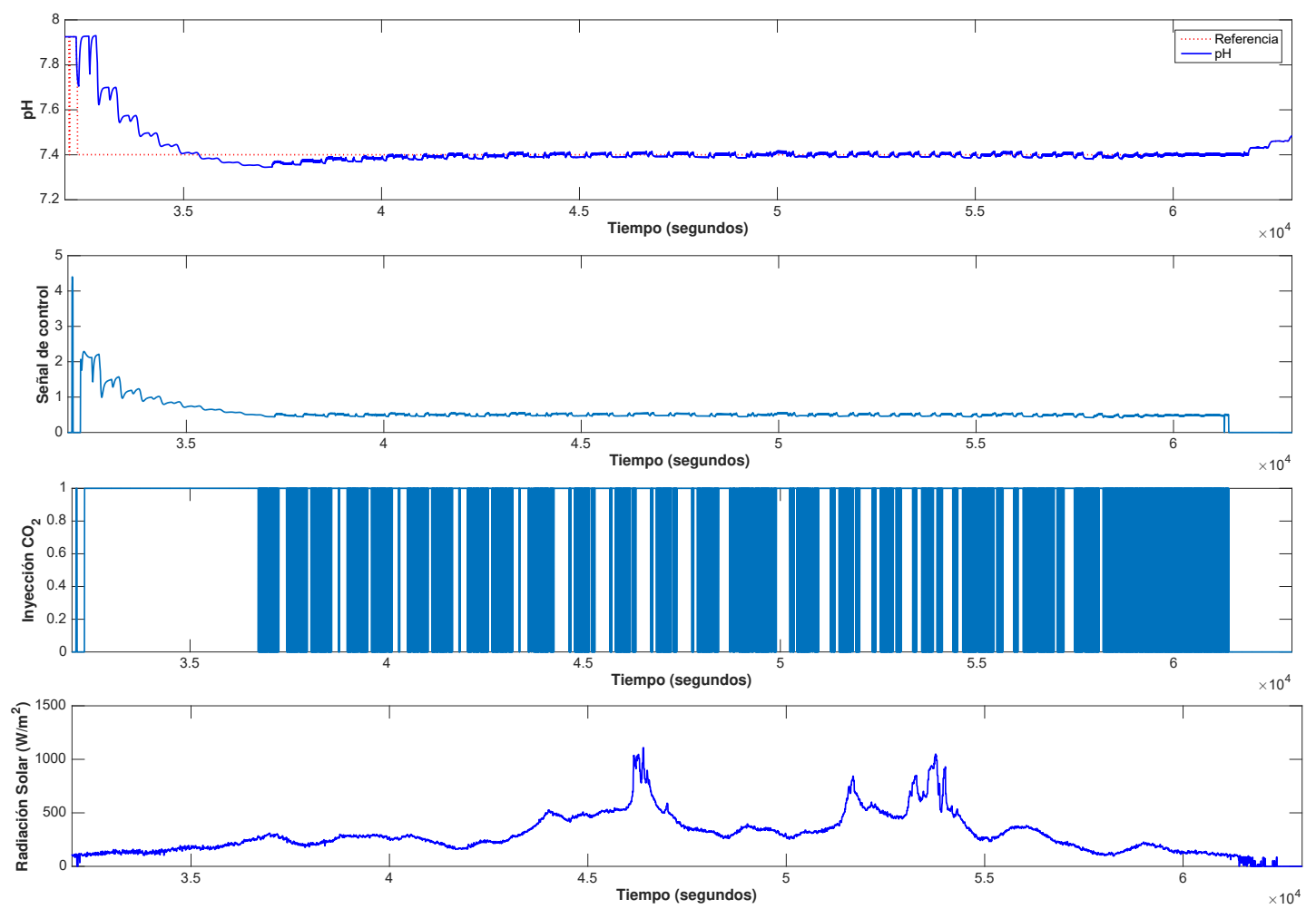

Figura 12: Ensayo día nublado para para un valor inicial de $\mathrm{pH}=7.9$ 
to. La señal de control hace referencia a la salida del controlador PID, mientras que la inyección de $\mathrm{CO}_{2}$ es la salida generada por el PWM.

\section{Conclusiones}

En este trabajo se ha presentado el control robusto con QFT del pH de un reactor tipo raceway. En primer lugar, la dinámica no lineal del proceso ha sido capturada en forma de modelo de bajo orden con incertidumbre paramétrica. El modelo ha sido obtenido tras realizar ensayos para 20 días diferentes y 3 puntos de operación cercanos a la zona de productividad óptima del proceso. Posteriormente, se procedió al diseño de un controlador robusto que fue evaluado en simulación para diferentes puntos de operación y bajo diversas condiciones climatológicas, obteniendo resultados satisfactorios. Como trabajos futuros se pretenden capturar igualmente la incertidumbre del término del modelo referente a la perturbación del sistema y realizar un control por adelanto robusto. Igualmente, se tiene planificado realizar ensayos reales en el reactor industrial.

\section{Agradecimientos}

Este trabajo ha sido financiado con el Proyecto del Plan Nacional DPI2014-55932-C2-1-R del Ministerio de Economía y Competitividad y Fondos FEDER.

\section{Referencias}

[1] J. Weissman and R. Goebel, "Design and analysis of pond system for the purpose of producing fuels," (Institute, Golden CO, SERI/STR.), 231st ed. Solar Energy Research., 1987.

[2] W. J. Oswald and C. G. Golueke, "Biological transformation of solar energy.," Appl. Microbiol., vol. 2, p. 223-262, 1960.

[3] C. Pawlowski, J. L. Mendoza, J. L. Guzmán, M. Berenguel, F. Acién, and S. Dormido, "Effective utilization of flue gases in raceway reactor with event-based ph control for microalgae culture," Bioresource Technology, vol. 170, pp. 1-9, 2014.

[4] T. A. Costache, F. G. Acién, J. M. Morales, M. M.and Fernández-Sevilla, I. Stamatin, and E. Molina, "Comprehensive model of microalgae photosynthesis rate as a function of culture conditions in photobioreactors," Applied Microbiology and Biotechnology, pp. 7627-7637, 2013.
[5] I. Fernández, F. G. Acién, J. L. Guzmán, M. Berenguel, and J. L. Mendoza, "Dynamic model of an industrial raceway reactor for microalgae production," Algal Research, vol. 17, pp. 67-78, 2016.

[6] A. Pawlowski, J. L. Mendoza, J. L. Guzmán, M. Berenguel, F. G. Acién, and S. Dormido, "Selective ph and dissolved oxygen control strategy for a raceway reactor within an event-based approach," Control Engineering Practice, vol. 44, pp. 209 - 218, 2015.

[7] I. Fernández, J. Peña, J. L. Guzman, M. Berenguel, and F. G. Acién, "Modelling and control issues of ph in tubular photobioreactors," IFAC Proceedings Volumes, vol. 43, no. 6 , pp. 186 - 191, 2010. 11th IFAC Symposium on Computer Applications in Biotechnology.

[8] A. Pawlowski, J. L. Mendoza, J. L. Guzmán, M. Berenguel, F. G. Acién, and S. Dormido, "Effective utilization of flue gases in raceway reactor with event-based ph control for microalgae culture," Bioresource Technology, vol. 170, pp. $1-9,2014$.

[9] J. L. Mendoza, I. Granados, M.R.and deGodos, F. G. Acién, E. Molina, C. J. Banks, and S. Heaven, "Fluid-dynamic characterization of real-scale raceway reactors for microalgae production.," Biomass and Bioenergy, vol. 54, p. 267-275, 2013.

[10] M. Berenguel, F. Rodríguez, and J. G. Acién, "Model predictive control of ph in tubular photobioreactors.," Journal of Process Control, vol. 14, p. 377-387, 2004.

[11] E. Sierra, F. Acién, J. Fernández, J. Garcíaa, C. González, and E. Molina, "Characterization of a flat plate photobioreactor for the production of microalgae.," Chemical Engineering Journal, vol. 138, pp. 136-147, 2008.

[12] I. M. Horowitz, Quantitative Feedback Design Theory (QFT). Boulder, Colorado: QFT Publications, 1993. 